\title{
Video Article \\ Driving Simulation in the Clinic: Testing Visual Exploratory Behavior in Daily Life Activities in Patients with Visual Field Defects
}

\author{
Johanna Hamel ${ }^{1,2}$, Antje Kraft ${ }^{1}$, Sven Ohl ${ }^{3}$, Sophie De Beukelaer ${ }^{1}$, Heinrich J. Audebert ${ }^{1,2}$, Stephan A. Brandt ${ }^{1}$ \\ ${ }^{1}$ Department of Neurology, Universitätsmedizin Charité \\ ${ }^{2}$ Center for Stroke Research Berlin (CSB), Universitätsmedizin Charité \\ ${ }^{3}$ Berlin School of Mind and Brain, Humboldt Universität zu Berlin
}

Correspondence to: Johanna Hamel at johanna.hamel@charite.de

URL: https://www.jove.com/video/4427

DOI: doi:10.3791/4427

Keywords: Medicine, Issue 67, Neuroscience, Physiology, Anatomy, Ophthalmology, compensatory oculomotor behavior, driving simulation, eye movements, homonymous hemianopia, stroke, visual field defects, visual field enlargement

Date Published: 9/18/2012

Citation: Hamel, J., Kraft, A., Ohl, S., De Beukelaer, S., Audebert, H.J., Brandt, S.A. Driving Simulation in the Clinic: Testing Visual Exploratory Behavior in Daily Life Activities in Patients with Visual Field Defects. J. Vis. Exp. (67), e4427, doi:10.3791/4427 (2012).

\section{Abstract}

Patients suffering from homonymous hemianopia after infarction of the posterior cerebral artery (PCA) report different degrees of constraint in daily life, despite similar visual deficits. We assume this could be due to variable development of compensatory strategies such as altered visual scanning behavior. Scanning compensatory therapy (SCT) is studied as part of the visual training after infarction next to vision restoration therapy. SCT consists of learning to make larger eye movements into the blind field enlarging the visual field of search, which has been proven to be the most useful strategy ${ }^{1}$, not only in natural search tasks but also in mastering daily life activities ${ }^{2}$. Nevertheless, in clinical routine it is difficult to identify individual levels and training effects of compensatory behavior, since it requires measurement of eye movements in a head unrestrained condition. Studies demonstrated that unrestrained head movements alter the visual exploratory behavior compared to a headrestrained laboratory condition ${ }^{3}$. Martin et al. ${ }^{4}$ and Hayhoe et al. ${ }^{5}$ showed that behavior demonstrated in a laboratory setting cannot be assigned easily to a natural condition. Hence, our goal was to develop a study set-up which uncovers different compensatory oculomotor strategies quickly in a realistic testing situation: Patients are tested in the clinical environment in a driving simulator. SILAB software (Wuerzburg Institute for Traffic Sciences GmbH (WIVW)) was used to program driving scenarios of varying complexity and recording the driver's performance. The software was combined with a head mounted infrared video pupil tracker, recording head- and eye-movements (EyeSeeCam, University of Munich Hospital, Clinical Neurosciences).

The positioning of the patient in the driving simulator and the positioning, adjustment and calibration of the camera is demonstrated. Typical performances of a patient with and without compensatory strategy and a healthy control are illustrated in this pilot study. Different oculomotor behaviors (frequency and amplitude of eye- and head-movements) are evaluated very quickly during the drive itself by dynamic overlay pictures indicating where the subjects gaze is located on the screen, and by analyzing the data. Compensatory gaze behavior in a patient leads to a driving performance comparable to a healthy control, while the performance of a patient without compensatory behavior is significantly worse. The data of eye- and head-movement-behavior as well as driving performance are discussed with respect to different oculomotor strategies and in a broader context with respect to possible training effects throughout the testing session and implications on rehabilitation potential.

\section{Video Link}

The video component of this article can be found at https://www.jove.com/video/4427/

\section{Protocol}

\section{Preparation of Study Position}

1. Let the patient take a seat, with distance of $2 \mathrm{~m}$ in front of the screen, $(203 \times 152 \mathrm{~cm}$ covering 58.15 degree of the visual angle on the horizontal axis and 43.61 degree of the visual angle on the vertical axis, resolution: $1400 \times 1050$ ), in a fixed base simulation car seat imitating a real car seat. Help the patient to adjust the seat distance to the pedal with the handle underneath. Help to adjust the backrest.

2. Instruct the patient how to use the simulation car gadgets (brakes, turn signal, steering wheel).

3. Instruct the patient in the task: Drive as you would do in a real non-simulated driving situation. The road is a one way single lane road with curves (smallest radius $500 \mathrm{~m}$, biggest radius $1,200 \mathrm{~m}$ ) and without traffic. Be vigilant to street signs and break-down cars emerging on both sides of the road. React to the notion of potentially hazardous events such as wild boar or balls approaching the road as soon as possible by either pressing the brake or using the turn-signal or both, what seems to be appropriate in the respective driving situation. While pressing the pedal, the car speeds up to a constant speed of $70 \mathrm{~km} / \mathrm{h}$ unless the brake is used ${ }^{1}$. The drive takes about $10 \mathrm{~min}$.

4. Inform the patient about simulation sickness. In case malaise, nausea or sweating occurs, interrupt testing session. 
5. A test drive with less task density is conducted to get used to the simulation situation and to prevent simulation sickness by allowing enough time to adjust ${ }^{2}$.

\section{Calibration of Eye Tracker}

1. In the second testing session, after the patient is properly seated and has received enough time for practice, place the Eye-Tracker on the patient's head and adjust it by pulling the flexible straps. The head camera laser should be pointing to the middle of the screen. Adjust the head camera's focusing on the pupil.

2. Instruct the patient to look at the five dots according to the lead of the mouse arrow for calibration.

3. Start the simulation.

4. Complete calibration with adding the horizontal calibration: Patient fixates overlay picture (of an eye) on screen on the left, then follows the eye moving across the screen and fixates it again on the right side.

5. Test the calibration by asking the patient to fixate on specific objects on the screen, and matching it with the overlay eye picture, which indicates the gaze position calculated by the software. The calibration is successful, if gaze and overlay picture meet at the same spot on screen. A vertical drift of the eye tracker acuity may occur during the drive. Evaluate the amount of drift by visual inspection at the beginning and at the end of the drive, checking the need for retesting.

6. If calibration was successful, turn off overlay pictures. If not repeat process of calibration until it is successful.

7. For quick assessment of compensatory behavior of gaze-movements turn on overlay eye pictures.

\section{Simulation}

1. Proceed with simulation by asking the patient to start driving.

2. Let the patient drive diverse routes (each a 6,500 $\mathrm{m}$ in rural areas and approximately 10 min duration) with different task difficulty due to level of distraction by surrounding environment. Each patient drives three routes.

3. Immediate assessment of oculomotor behavior: Turn on the overlay eye pictures and visualize the gaze behavior of a patient during testing session: The eye-tracker continuously sends coordinates of the actual gaze position to the simulation software SILAB. In return SILAB projects the overlay eye picture, which is an image of an eye, on the screen exactly on the spot where the patient looks at. This can not only be used to prove quality of calibration, but also to make gaze behavior immediately visible not only to you but also to the patient.

\section{Analysis}

1. For data recording use SILAB Software at a sample rate of $100 \mathrm{~Hz}$. Use SILAB Software also to record speed, reaction times (use of turn signal, brake).

2. Perform statistical analysis of head- and eye-movement parameters with Matlab (MathWorks Company, Natick, USA). Use the following criteria:

1. Define saccades as sections of the gaze trajectory where gaze velocity exceeds $30^{\circ} / \mathrm{s}$ and gaze amplitude is larger than $1^{\circ}$ (as eye movements below $1^{\circ}$ belong to microsaccades). Cluster saccades occurring within $80 \mathrm{~ms}$. Define sections between saccades as fixations. Define head-movements as movements exceeding $6^{\circ} / \mathrm{sec}^{11}$ and an amplitude of more than $3^{\circ}$. Exclude simultaneous headand eye-movements with directory in the opposite direction as they represent no gain in gaze amplitude.

2. Define object fixations as fixation on an object with gaze position maximal $1,24^{\circ}$ apart from the object on the $x$-axis and $1,66^{\circ}$ on the $y$ axis. Objects are not triggered according to the patients gaze position, but consider eccentricity of object to gaze position by calculating it when the object appears ${ }^{3}$.

3. Calculate the average length of the participants' fixations (mean fixation durations) and the spread of search in the horizontal and vertical meridians (the variance of the fixation locations).

3. Measure reaction times in two ways: As a first mode (first detection) measure reaction time as first detection by either fixation or manual detection: If the patient fixates the object first and responds manually afterward (in the majority of cases), then chose the fixation time as reaction time as first detection. If the patient uses the turn signal or brake pedal first as an indicator without first fixating the object, then chose manual reaction time as first detection. As a second mode (manual reaction), measure reaction time by manual reaction (brake or turn signal) only.

\section{Representative Results}

We recruited 6 patients of different ages (35-71 years of age) with incomplete hemianopia after ischemic PCA infarction (4 on the right and 2 on the left hemisphere) and 85 healthy controls of various ages (20-75 years of age, equally distributed) to determine age related changes in eye- and head-movements as well as driving performance as a reference group. They did not report cognitive deficits, neurological or psychiatric deficits or diseases and visual acuity was higher than 0.5 . The medical history was taken and experiences with virtual media explored. The study was conducted in conformity with the declaration of Helsinki and was approved by the local ethics committee. Written informed consent was obtained from all participants. All subjects were unaware of the purpose of the experiments.

Here, we demonstrate representative test results of two patients tested 7- 9 months after stroke incident with incomplete hemianopia (Figure 1) on the right side with and without compensatory behavior as well as a healthy subject as a control. The healthy control was chosen due to similar age, driving and computer game experience.

Patient A exhibited compensatory saccadic movement to the side where the visual defect is located resulting in normal performance in driving simulation compared to a healthy control with successful detection of and reaction to possible hazards in a rural driving situation. However, patient $B$ did not show compensatory saccadic movement and revealed poor performance in driving simulation with missing out on peripheral objects in the blind field causing prolonged reaction times or collisions. Nevertheless, throughout the drives, patient B adopted compensatory 
behavior causing fewer collisions, without being instructed to do so. The testing was performed head-unrestrained allowing realistic conditions and to detect the possible influence of head movements in compensatory behavior.

The patients were asked to drive the course as they would do in a real non-simulated driving situation. Compared to the healthy subject patient $A$ performed saccades 1.7 times more frequently, which predominantly covered the side of the screen where the visual defect was located (63\%). The amplitudes of the saccades in Patient $A$ and the control were similar (mean amplitude: 5.5 degree in the healthy subject versus 5.3 degree in the patient). The fixation duration of patient $A$ was shorter compared to the healthy control (mean fixation duration of 381 ms in patient $A$ versus $483 \mathrm{~ms}$ in the control).

In contrast patient $B$ and the control explored equally frequent both sides of the screen. Figure 2 illustrates the distribution of fixations on the screen during the first drive of patient $A$, the healthy subject and patient $B$. Patient $B$ performed 3.4 less saccadic movements compared to patient $A$ covering half the size of the amplitude of patient $A$ (mean amplitude: 5.5 patient $A$ versus 2.9 degree in patient $B$ ). Patient $B$ showed longer fixation durations compared to both healthy control and patient $A$ (mean fixation duration $1049 \mathrm{~ms}$ ).

Patient A and patient B performed almost no head-movements (1 to 2 ) while the healthy control executed a few (5 to 10) head-movements per driving session contributing to gaze amplitude.

Figure 3 demonstrates the influence of eccentricity of object position with respect to gaze position on the reaction time, demonstrated separately for the left and right side of the visual field. The figure illustrates the increase of reaction time due to eccentricity in the two subjects illustrated separately for both sides of the visual field. Some reaction times at very small eccentricities are less than 50 ms. These are not realistic reaction times but rather due to scanning of possible hazardous locations along the road, or objects appearing at the point of fixation of the patient. We did not filter those events because this also represents a certain driving behavior of interest: acknowledging and yielding to potential hazardous locations. (The graphic also shows that there were less reaction times noted for patient B due to missed objects in his blind field.)

In patient $A$ and the healthy control all objects were detected and no collisions occurred. In patient $B$ though, reaction times differed clearly between right (blind) and left (sighted) field: Patient B detected objects occurring in the blind field 1.6 times slower compared to the sighted field and collided 4 times with objects occurring in the blind field (median reaction times: right (blind) field: 4411.66 ms versus left (sighted) field: 2810 $\mathrm{ms})$.

Hence, patient A compensated his loss of right eccentric vision well by an increased number of saccadic movements reaching the side of the visual field defect. It is still unclear though if this compensatory strategy becomes insufficient with higher workload. Evidence for this is suggested in the graph for the left visual field: While the patient managed to react equally fast on the right side due to lateralization of saccadic movements, he showed longer reaction times at greater eccentricities on the left side, suggesting a possible cost of the strategy with regard to performance. However, the healthy control also shows slight differences of reaction times comparing both sides, which might also be due to the fact that the healthy control performed one drive less than the patients. To test whether this represents a stable effect, more trials would be needed.

In contrast to patient A, patient B presented a representative outcome of a patient lacking compensatory behavior and its effect on driving performance: the lack of compensatory saccadic movements into the blind field resulted in collision with objects appearing in the blind field and prolonged reaction times. Nevertheless, throughout the drives, the patient spontaneously started to perform more saccades into the right visual field with greater amplitude, resulting in smaller incidence of collisions.
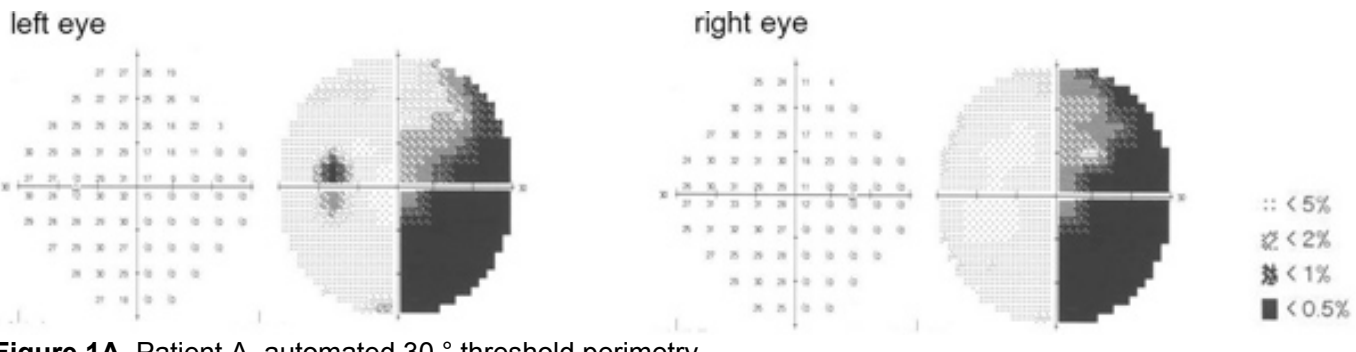

Figure 1A. Patient $A$, automated $30^{\circ}$ threshold perimetry.
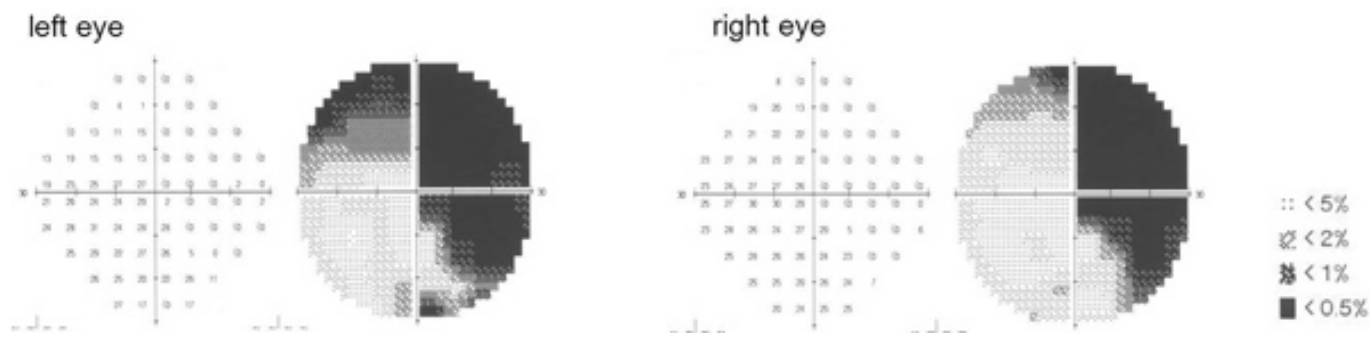

Figure 1B. Patient $B$, automated $30^{\circ}$ threshold perimetry. 


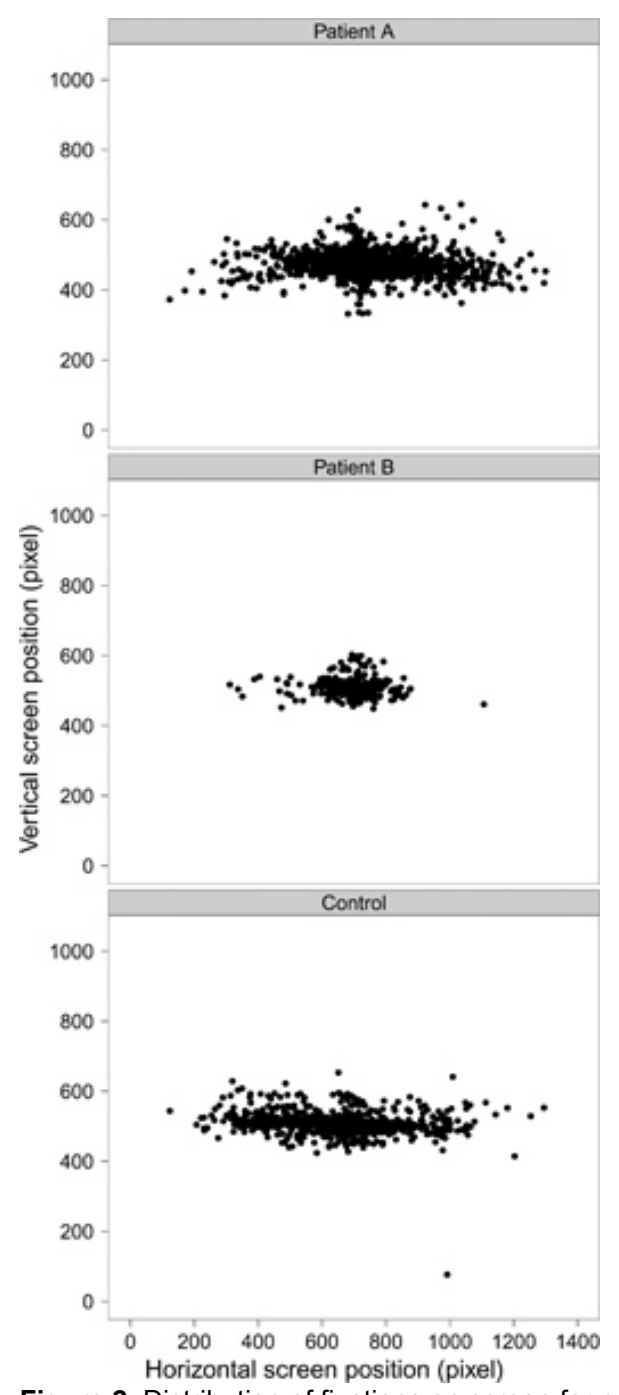

Figure 2. Distribution of fixations on screen for patient A, patient $B$, and healthy control. 


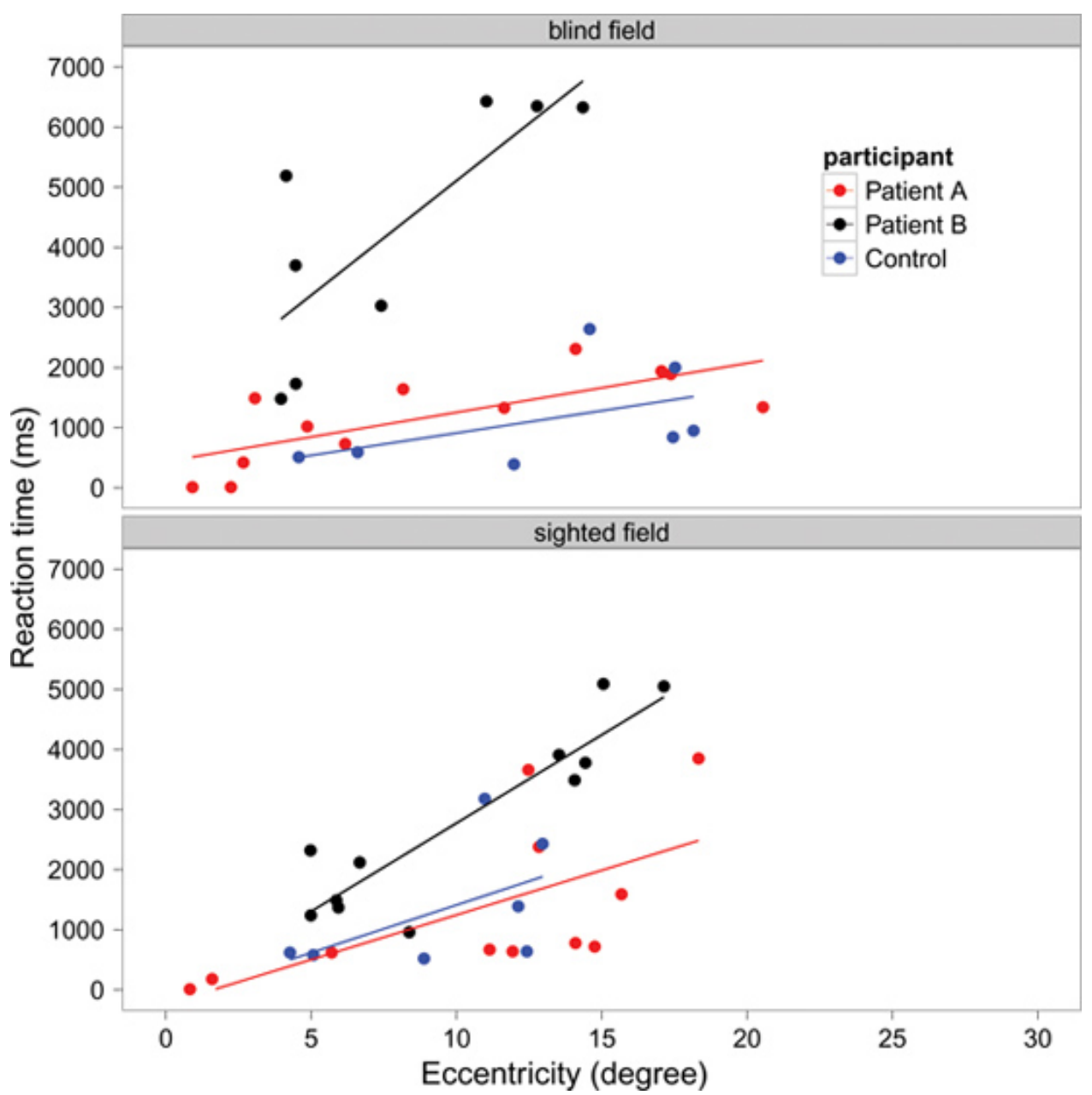

Figure 3. Reaction times to objects appearing at various eccentricities in the visual field, for patient $A$, patient $B$, and healthy control.

${ }^{1}$ This tempomat was implemented to assure comparability of reaction times between age groups as it is known that older drivers reduce speed as a possible compensatory mechanism ${ }^{7}$.

${ }^{2}$ Simulation sickness is described as nausea, sweating or dizziness persisting during a driving session. There is different data on frequency of occurrence ranging from $9 \%$ to $37 \%$ depending on age as it occurs more likely in the elderly ${ }^{8,9,10}$. Thorough preparation with practice drives long enough for each individual for proper adjustment reduce the chance of simulation sickness.

${ }^{3}$ Per drive there are 4 wild boar and 4 balls programmed to approach from each side of the road at two different eccentricities, at straight parts of the course and at different intervals of the course to prevent a testing habit. Appearance of objects is triggered by the subject passing flow points on the road.

\section{Discussion}

The new established method enables the examination of visual exploratory behavior of patients with visual field defects caused by a stroke. The test design also offers an immediate approach to evaluate compensatory gaze behavior: By turning on the overlay eye pictures the examiner can visualize the gaze behavior of a patient during testing session. Hence, it allows a very quick and immediate assessment as to whether the patient has adopted a compensatory gaze behavior. It also allows patients to become aware of it by visualizing gaze movements by an overlay eye picture moving across the screen as a gaze indicating feedback tool. The role of head-movements in compensatory gaze behavior is still unclear. In our control group head-movements were more common among the elderly. The healthy control performed more head-movements than the patients. Head-movements may play a bigger role when the field of view tested is wider than in our set-up. Hence we could not identify head-movements as part of compensatory gaze behavior in our patient. However more patients need to be examined to clarify the role of headmovements in compensatory behavior.

Limitations of the study are the following: Retesting becomes necessary in some individuals due to vertical drift of the eye tracker throughout the drive. Objects appear naturally along the road and not at a fixed eccentricity triggered by the gaze position. Nevertheless current gaze position in relation to the object is considered when interpreting reaction times.

Patients with visual field defects have been tested before in simulated and real driving settings: Bowers et al. ${ }^{12}$ and Cockelbergh et al. ${ }^{13}$ carried out studies in a driving simulator and demonstrated poorer driving performance in patients compared to healthy controls. However they did not record eye- and head-movements and individual differences could not be related to visual exploratory behavior. Wood et al. ${ }^{6}$ tested in a 
real life situation and established an evaluation of driving performance of patients with visual field defects. Head- and eye-movements were analyzed via video and post-test scoring by two independent researchers, thus dealing with inter-rater-reliability. Nevertheless they did not provide a quantitative analysis of fixation durations, saccades, and head movements and evaluation depended on a certified driving rehabilitation specialist. The advantage of our set up with simulated driving is the easy and fast assessment within a clinical setting, recording of well-defined parameter of eye- and head- movements as well as reaction times. It is possible to control the level of distraction and expose each driver to a similar driving situation with standardized routes and conditions allowing comparability. Roth ${ }^{2}$ has shown that SCT improves search behavior on the blind sight in natural search tasks. By adjusting the level of distraction in the driving courses, it will be possible to prove if, and at what level, the compensatory behavior fails with higher workload. Comparing simulated to real driving studies, it seems appropriate to teach compensatory behavior in a simulated environment and expose the patient to a real driving situation as a second step. Especially since the latter enables to evaluate safety of driving.

In the future we intend to include characterization of different levels of compensatory behavior by analyzing saccades, amplitudes and distribution. This could help to offer more individual rehabilitation plans adjusted to the patient's current level of compensatory behavior. Secondly, as patient $B$ reveals spontaneous adoption of a compensatory strategy, we like to test the design as a possible tool for rehabilitation purposes: Driving simulation not just as a diagnostic test design but also for specific training, instructing the patient to perform compensatory saccade behavior. Combined with immediate visualization of gaze behavior by the gaze indicating eye overlay pictures this might provide a feedback mechanism to arise the attention to a compensatory strategy.

\section{Disclosures}

No conflicts of interest declared.

\section{Acknowledgements}

The study receives funding from the Federal Ministry of Education (BMBF) via the grant CSB (01 EO 0801). The Center for Stroke Research Berlin (CSB) is an integrated research and treatment centre. We thank the Stiftung Felgenhauer for financial support.

We thank Richard A. Dargie for corrections to the English text.

\section{References}

1. Bouwmeester, L., Heutink, J., \& Lucas, C. The effect of visual training for patients with visual field defects due to brain damage: a systematic review. Journal of Neurology, Neurosurgery, and Psychiatry. 78 (6), 555-64 (2007).

2. Roth, T., Sokolov, A.N., Messias, A., Roth, P., Weller, M., \& Trauzettel-Klosinski, S. Comparing explorative saccade and flicker training in hemianopia. A randomized controlled study. Neurology. 72, 324-331 (2009).

3. Freedman, E.G. Coordination of the eyes and head during visual orienting. Experimental brain research. 190 (4), $369-87$ (2008).

4. Martin, T., Riley, M.E., Kelly, K.N., Hayhoe, M., \& Huxlin, K.R. Visually-guided behavior of homonymous hemianopes in a naturalistic task. Vision Research. 47 (28), 3434-46 (2007).

5. Hayhoe, M.M. \& Ballard, D. Eye movements in natural behavior. Trends in Cognitive Sciences. 9, 188-194 (2005).

6. Wood, J.M., McGwin, G., Elgin, J., et al. Hemianopic and quadrantanopic field loss, eye and head movements, and driving. Investigative ophthalmology \& visual science. 52 (3), 1220-5 (2011).

7. Cantin, V., Lavalli re, M., Simoneau, M., \& Teasdale, N. Mental workload when driving in a simulator: effects of age and driving complexity. Accident; analysis and prevention. 41 (4), 763-71 (2009).

8. Brooks, J.O., et al. Simulator sickness during driving simulation studies. Accident; analysis and prevention. 42 (3), $788-96$ (2010).

9. Allen, R.W., Park, G.D., Fiorentino, D., Rosenthal, T.J., \& Cook, L.M. Analysis of simulator sickness as a function of age and gender. $9^{\text {th }}$ Annual Driving Simulation Conference Europe Paris, France., (2006).

10. Liu, L., Watson, B., \& Miyazaki, M. VR for the Elderly: Quantitative and Qualitative Differences in Performance with a Driving Simulator. Cyberpsychol. Behav. 2 (6), 567-577 (1999).

11. Einhäuser, W., Moeller, G.U., Schumann, F., Conradt, J., Vockeroth, J., Bartl, K., Schneider, E., \& König, P. Eye-Head Coordination during Free Exploration in Human and Cat. Basic and Clinical Aspects of Vertigo and Dizziness., Ann. N. Y. Acad. Sci. 1164, 353-366 (2009).

12. Bowers, A.R., Mandel, A.J., Goldstein, R.B., \& Peli, E. Driving with Hemianopia, I: Detection Performance in a Driving Simulator. Investigative Ophthalmology and Visual Science. 50, 5137-5147 (2009).

13. Cockelbergh, T.R.M., Brouwer, W.H., Cornelissen, F.W., van Wolffelaar, P., \& Kooijman, A.C. The Effect of Visual Field Defects on Driving Performance. Archives of Ophthalmology. 120, 1509-1516 (2002). 